\title{
Kinematic and kinetic study of sit-to-stand and stand-to-sit movements towards a human-like skeletal model*
}

\author{
Cristiana Pinheiro, João M. Lopes, Luís Moreira, Daniel Sanz-Merodio, Joana Figueiredo, Cristina P. \\ Santos, Elena Garcia
}

\begin{abstract}
The movements of sit-to-stand and stand-to-sit are frequently executed on daily life. To develop robotic assistance devices for people with mobility problems, it is important to study how a healthy human performs these tasks. The goal of this study is to present a mathematical model based on acquired kinematic and kinetic data that represents a healthy human body performing these movements. The results revealed that the movements of sit-to-stand and stand-to-sit have symmetric joint angles, torques and ground reaction force. The joint angles and the torques are very similar on each leg. The knee and the hip show more variation of the angle and achieve higher values of torque in comparison to the ankle. Although, ankle has higher torque during standing position. The ground reaction force show that is necessary create a force additional to the standard weight force to achieve a more stable position that is sitting or standing. The acquired data describe as expected the sit-to-stand and stand-to-sit movements and can be used to validate the presented model.
\end{abstract}

\section{INTRODUCTION}

Standing up from a chair and sitting down on a chair are indispensable activities of healthy daily living [1]. The inability to do these two tasks causes a dependent life and can lead to dead [1]. The safe execution of these movements requires a coordinated activation of several muscle groups and balance, creating joint torques greater than other daily activity [2,3]. Because of that, they are difficult or impossible activities for persons who have neuromuscular or/and musculoskeletal dysfunctions [3].

The sit-to-stand and stand-to-sit movements are symmetric movements [4] and, according to [5], they can be divided into two phases. In the first one, there is two contacts between the subject and the environment, which are the contact between the pelvis and the chair and the contact between the feet and the ground. In the second phase, the subject only has contact with the ground. In order to study

*This work has been supported in part by the Fundação para a Ciência e Tecnologia (FCT) with the Reference Scholarship under Grant SFRH/BD/108309/2015, and part by the FEDER Funds through the Programa Operacional Regional do Norte and national funds from FCT with the project SmartOs -Controlo Inteligente de um Sistema Ortótico Ativo e Autónomo- under Grant NORTE-01-0145-FEDER-030386, and by the FEDER Funds through the COMPETE 2020-Programa Operacional Competitividade e Internacionalização (POCI) - with the Reference Project under Grant POCI-01-0145-FEDER-006941.

C. Pinheiro, J. Figueiredo, and C. P. Santos are with the Center for MicroElectroMechanical Systems (CMEMS), University of Minho, Portugal (e-mail: \{a73727@alunos, id6003@alunos, cristina@dei\}.uminho.pt).

D. Sanz-Merodio is with Marsi Bionics, Spain (e-mail: daniel.sanz@marsibionics.com).

E. Garcia is with Center of Automation and Robotics (CAR) of Spanish National Research Council (CSIC), Spain (e-mail: elena.garcia@csic.es). these movements, the simplest and most widely used representation of the human body is through a skeletal model with three segments (i.e. shanks, thighs and HAT (HeadArms-Trunk)) and three degrees of freedom in respect to motion [2]. This skeletal model can be used to study the trajectory of the mass center of the body during these movements or to study the torques in the ankle, knee and hip joints through kinematic data [1].

The goal of this study is to present a mathematical model that represents the movements of sit-to-stand and stand-to-sit in healthy adults. The model is based on principles of Lagrange's dynamics. It considers the hip, knee and ankle joints and the HAT, thighs and shanks segments in the sagittal plane. To validate the model, kinematic and kinetic data were collected from 14 healthy adults at a selfcomfortable speed, using an optoelectronic motion capture system and force plates, respectively. In this paper, the mean and the standard deviation, in respect to all subjects, of the ankle, knee and hip angles and torques, and the ground reaction force will be presented and analysed. In the future, this model can be used in the development of robotic assistive devices which can improve life quality of persons who have not the ability to do these tasks [3].

\section{HUMAN-LIKE SKELETAL MODEL}

The proposed skeletal model includes three segments with three degrees of freedom in respect to the sit-to-stand and stand-to-sit movements, for sagittal plane. Because it is assumed that there is a symmetry of body when it does these movements [3], the shanks of the two legs are represented in one segment and the thighs of the two legs are, also, represented in one segment. The remained segment is the HAT, which combines the head, arms and trunk. Each segment is considered as a rigid body with its mass contained in its center of mass. The skeletal model motion is described by (1) which comes from Lagrange's dynamic [6], where $K$ is the sum of the kinetic energy of each segment; $P$ is the sum of the potential energy of each segment; $\theta_{i}$ is the angular variation of the segment $i$ in relation to vertical for $i=1$ or in relation to the distal segment for $i=2,3$ during the movement; $t$ is time; $W$ is the total work done by the applied moments.

$$
\frac{d}{d t}\left(\frac{\partial L}{\partial \dot{\theta}_{i}}\right)-\frac{\partial L}{\partial \theta_{i}}=\frac{\partial W}{\partial \theta_{i}}, i=1,2,3 \text { and } L=K-P
$$

The Fig. 1 illustrates the model. The segments 1, 2 and 3 represent the two shanks, the two thighs and the HAT, respectively, and the joints 1, 2 and 3 represent the ankle, knee and hip joints, respectively. The model joints are 
considered as ideal joints because it is not considered the

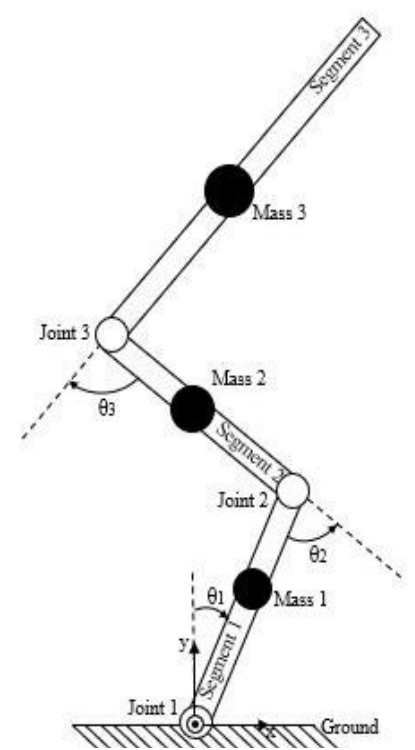

Figure 1. Skeletal model of human body.

friction during the rotation of segments.

The potential energy of one segment is defined as the product of the mass of the segment, the gravity acceleration constant and the height of the segment's mass center.

The kinetic energy of one segment is defined as (2), where $I$ is the segment's moment of inertia due rotation on $\mathrm{Z}$ axis, $m$ is the mass of the segment and $v$ is the linear velocity of the segment's mass center. The linear velocity is defined as $\dot{\theta} d+v^{*}$, where $d$ is the length between the distal joint and the segment's mass center and $v^{*}$ is the linear velocity of the joint. The linear velocity of the joint 1 is considered null during all the movement because the feet do not move during all the movement. For the remained joints, the linear velocity is calculated through the same expression but $\dot{\theta}$ is the angular velocity of the distal segment, $\mathrm{d}$ is the length to the distal joint and $\mathrm{v}^{*}$ is the linear velocity of the distal joint.

$$
K_{i}=0.5\left(\dot{\theta}_{i}^{2} I_{i}+m_{i} v_{i}^{2}\right), i=1,2,3
$$

According to [7], the mass of each segment and the length between the mass center and the distal joint can be obtained through the body mass and the segment's length, respectively. In addition, according to [7], the inertia can be obtained from the segment's mass and its turning radius.

In this manner, the inputs of the model are kinematic and anthropometric data. The kinematic data are angle, angular velocity and angular acceleration of each joint during the movement. Regarding the anthropometric data, the body mass is included and the length of each segment of the user. The model yields the torque of each joint.

\section{DATA ACQUISITION}

\section{A. Participants}

This study includes 14 healthy subjects [mean (standard deviation): age 30 (7) years, height 1.717 (0.087) m, body mass $70.2(12.0) \mathrm{kg}$. The subjects filled in and signed a medical questionnaire to verify their eligibility and an informed consent form to participate in this study. The eligibility was achieved if the subject did not have neuromuscular and musculoskeletal impairment.

\section{B. Materials}

It was used an optoelectronic motion capture system (Vicon, United Kingdom) with 8 infrared cameras (Vicon Vero, United Kingdom) and two ground-levelled force plates (Kistler, Switzerland) allocated side by side to acquire kinematic and kinetic data, respectively.

Fig. 2 shows the seat that was used. It had not backrest to prevent upper limb involvement and markers occlusion and the armrest was not used for the same reason. One of the seven height levels was chosen for each subject in order to all have the same initial position in sit-to-stand. In this manner, the initial position did not influence the results. The seat had a fixed position that was close to the force plates to allow each foot to be placed on each force plate.

\section{Subject Preparation}

In order to the motion capture system identify the subject motion, it was necessary place infrared reflective markers onto participant's body. In this experiment were attached 16 markers (each marker with $14 \mathrm{~mm}$ of diameter) by using double sided adhesive tape according to [8]. The locations of the markers were: left/right anterior superior iliac spine, left/right posterior superior iliac spine, over the lower/upper lateral $1 / 3$ surface of the left/right thigh, right/left knee, over the lower/upper lateral $1 / 3$ surface of the left/right shank, right/left ankle, right/left toe and right/left heel. The toe markers were allocated over the second metatarsal head, on the mid-foot side of the equinus break between fore-foot and mid-foot. Fig. 2 shows this marker set. In the experimental test, it was verified that the left and right anterior superior iliac spine markers were occluded by the body. In order to avoid this situation, they were moved laterally by an equal amount along the anterior superior iliac spine axis and the value of the distance between the left and right anterior superior iliac spine was introduced on the software.

Subsequently, the height of the seat was adjusted to the thigh be parallel to the ground in the seated position. Also, each foot was fully placed on each force plate with comfortable distance between them and was ensured that they made $90^{\circ}$ with the shank.

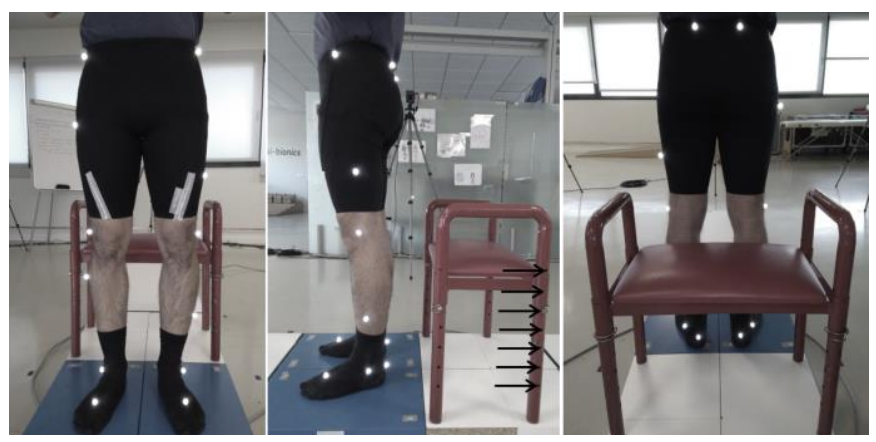

Figure 2. Photograph of the markers placed on the subject and the seat used in the experimental test. 


\section{Experimental test}

The data acquisition was made with a sampling frequency of $100 \mathrm{~Hz}$ and started with the subject seated during $3 \mathrm{~s}$. Then, the subject was asked to stand up and stood up during $3 \mathrm{~s}$ to ensure that the sit-to-stand movement was completed. After, the subject was asked to sit down and remained seated during $3 \mathrm{~s}$ to ensure that the stand-to-sit movement was completed. This procedure was done 4 times at a self-comfortable speed. The data acquisition ended with the subject seated during $3 \mathrm{~s}$. The subject was asked to look forward, to cross the arms on the chest to prevent arms involvement and to not move the feet.

\section{E. Data processing}

It was used a software (Vicon Nexus, Version 2.7.1, United Kingdom) to compute the angles and the torques of the hip, knee and ankle joints. These data were filtered at 5 $\mathrm{Hz}$ with a zero-lag low-pass Butterworth filter of $4^{\text {th }}$ order. The ground reaction force data provided by the force plates were also filtered at $5 \mathrm{~Hz}$ with a zero-lag low-pass Butterworth filter of $4^{\text {th }}$ order. The seat-off event, that occurs when the subject loses/makes contact with the chair in sit-tostand/stand-to-sit movement, was identified as the first time that the second derivative of the ground reaction force goes from negative to positive in sit-to-stand and as the second time that the second derivative of the ground reaction force goes from negative to positive in stand-to-sit [9]. It was necessary because the torques before that time could not be calculated once the contact between the subject and the seat could not be measured.

It was calculated the mean and standard deviation of the data from the 4 trials for each subject. Before this process, it was made a cubic interpolation and, then, a resampling, in order to the 4 trials have the same number of samples, because the duration of the movement varies between trials of the same subject. The number of samples chosen corresponds to the trial when the subject was faster. Subsequently, it was calculated the mean and standard deviation of the data from all the subjects through the mean of each subject in the 4 trials. Because the duration of the movement varies between subjects, before this process, it was made a cubic interpolation and resampling too and the number of samples chosen corresponds to the subject who executed faster the movement.

\section{RESUlTS AND DisCUSSION}

The Fig. 3 shows the angle and the torque for the ankle, knee and hip joints, in sagittal plane, during the execution of the sit-to-stand movement. The torque is normalized by the user's weight and height. The $\mathrm{x}$ axis shows the phase of the motion in percentage being that the $0 \%$ corresponds to the time after the seat-off event and the $100 \%$ corresponds the time when the subject was in standing position during $1 \mathrm{~s}$. The Fig. 4 shows the same parameters for the movement of standto-sit. In this case, the $0 \%$ is when the subject was in standing position during $2 \mathrm{~s}$ and the $100 \%$ corresponds to the time before the seat-off event. So, in the two movements is shown the subject in standing position during $1 \mathrm{~s}$ because the remained $2 \mathrm{~s}$ give the same conclusions. These data are according to the data presented in [4].
By analyzing Fig. 3 and Fig. 4, the sit-to-stand and standto-sit movements can be considered as symmetric movements in relation to the angular variation and the torques of the ankle, knee and hip joints. Besides that, the right and the left legs have the same pattern and the values are close in each movement. So, in healthy adults, the angular variation and the torques of the ankle, knee and hip joints in one leg can be consider to the other leg with a maximum difference in module of approximately $2 \mathrm{deg}$ and $0.05 \mathrm{~N} \cdot \mathrm{m} /(\mathrm{kg} \cdot \mathrm{m})$. Also, it is noticed that there is the same pattern for all the subjects with a maximum variability in module of approximately 20 deg and $0.2 \mathrm{~N} \cdot \mathrm{m} /(\mathrm{kg} \cdot \mathrm{m})$. In the sit-to-stand movement, after the seat-off event, the foot achieved maximum dorsiflexion and then occurs plantar flexion, decreasing the ankle angle from approximately $20 \mathrm{deg}$ to $5 \mathrm{deg}$. The knee and the hip joints extend from the maximum flexion with approximately $90 \mathrm{deg}$ and $80 \mathrm{deg}$, respectively, to approximately $7 \mathrm{deg}$ and $0 \mathrm{deg}$, respectively. Relatively to the joint torques, the knee and hip joints achieve the maximum value of approximately $0.47 \mathrm{~N} \cdot \mathrm{m} /(\mathrm{kg} \cdot \mathrm{m})$ and $0.43 \mathrm{~N} \cdot \mathrm{m} /(\mathrm{kg} \cdot \mathrm{m})$, respectively, and then decrease to approximately $0 \mathrm{~N} \cdot \mathrm{m} /(\mathrm{kg} \cdot \mathrm{m})$ and 0.03 $\mathrm{N} \cdot \mathrm{m} /(\mathrm{kg} \cdot \mathrm{m})$, respectively. On the other hand, the ankle
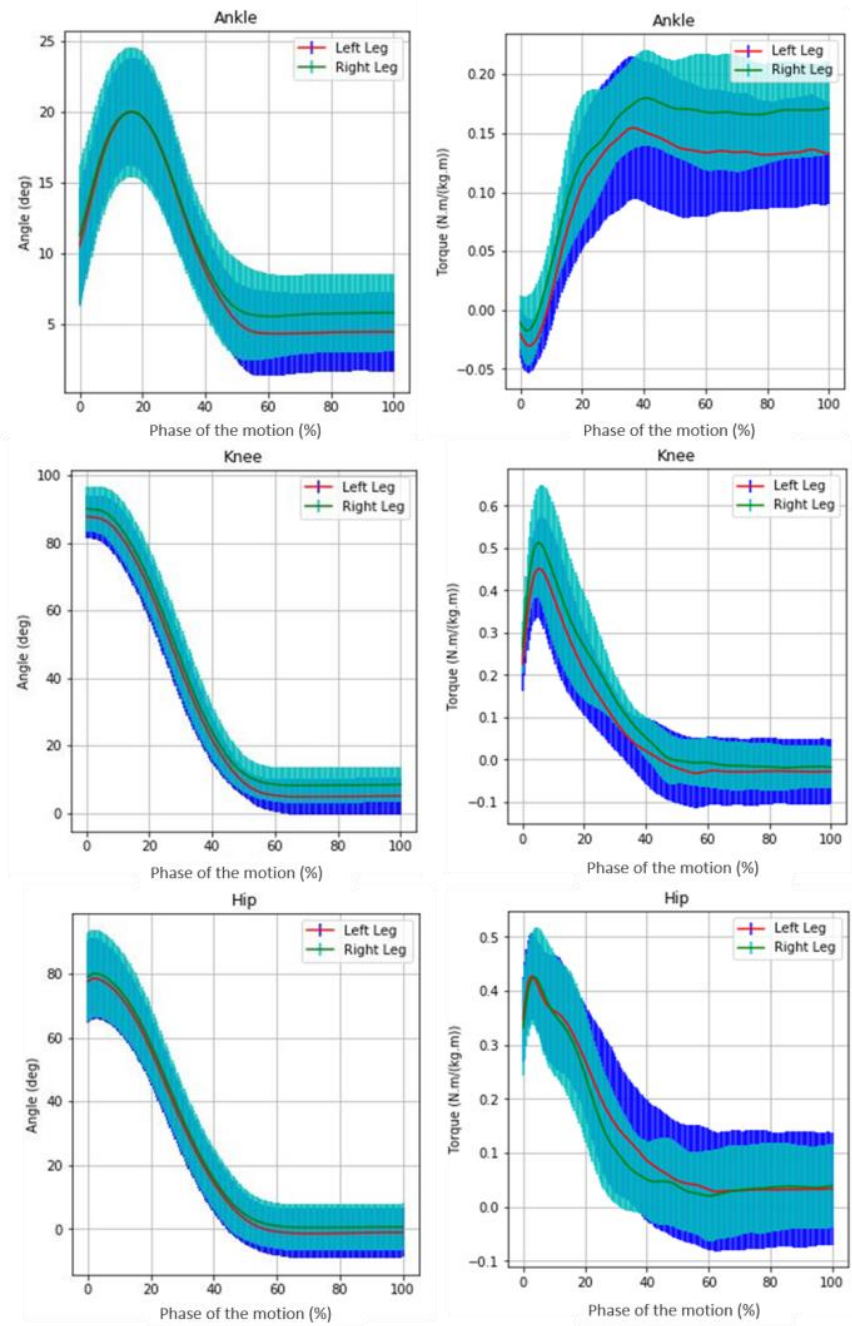

Figure 3. Mean and standard deviation of angle $(\mathrm{deg})$ and torque $\mathrm{N} \cdot \mathrm{m} /(\mathrm{kg} \cdot \mathrm{m})$ for the ankle, knee and hip joints during the execution of sit-to-stand movement $(\%)$ in respect to all subjects. The red line corresponds to the mean in relation to the left leg and the standard deviation is shown in dark blue. The green line corresponds to the mean in relation to the right leg and the standard deviation is shown in light blue. 

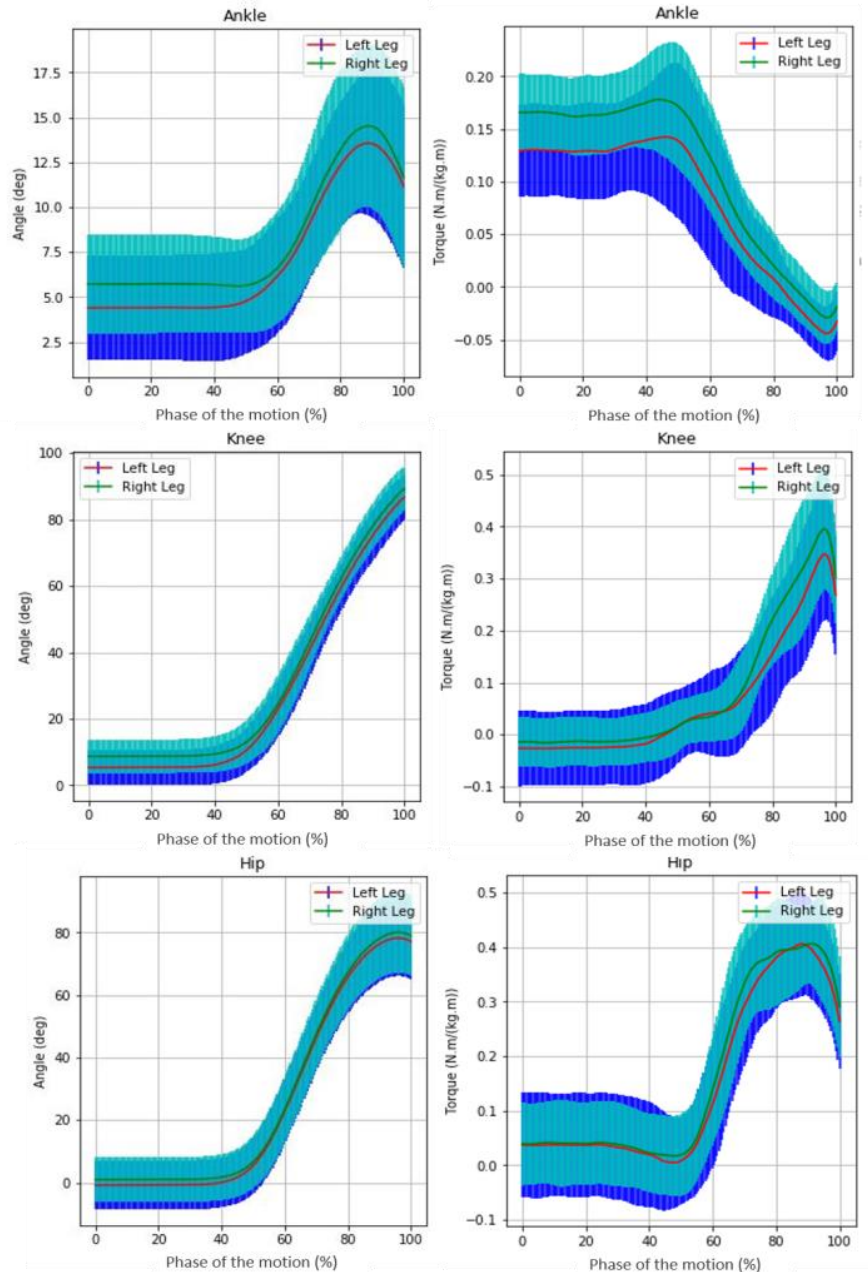

Figure 4. Mean and standard deviation of angle (deg) and torque $\mathrm{N} \cdot \mathrm{m} / \mathrm{kg} \cdot \mathrm{m})$ for the ankle, knee and hip joints during the execution of stand-to-sit movement (\%) in respect to all subjects. The red line corresponds to the mean in relation to the left leg and the standard deviation is shown in dark blue. The green line corresponds to the mean in relation to the right leg and the standard deviation is shown in light blue.

torque achieves the minimum value that is approximately $0.025 \mathrm{~N} \cdot \mathrm{m} /(\mathrm{kg} \cdot \mathrm{m})$ and increase to approximately 0.15 $\mathrm{N} \cdot \mathrm{m} /(\mathrm{kg} \cdot \mathrm{m})$. So, the hip and the knee joints have higher torque in comparison with the ankle joint in these movements. Although, the ankle joint has a higher value of torque in standing position in comparison with the hip and knee joints.

The Fig. 5 and the Fig. 6 show the vertical ground reaction force normalized by weight to the movements of sitto-stand and stand-to-sit, respectively. These data are according to the data presented in [9].

Through the analysis of Fig. 5 and Fig. 6, it can be deduced that, when the subject is in stable standing position, the normalized ground reaction force corresponds to the gravity acceleration constant. When the subject stands up from the seat creates a force additional to the standard weight force and, because of that, the value of the ground reaction force is, in module, higher than the gravity acceleration constant. Then, the subject moves to a more stable position and the additional force decrease. This decreasing makes the normalized ground reaction force be lower than the gravity acceleration constant. So, during this period, the standard weight force is not all applied on the ground. These can be

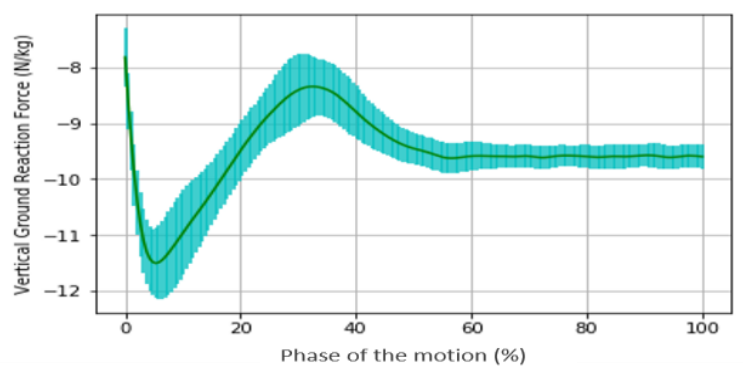

Figure 5. Mean (green line) and standard deviation (light blue) of normalized vertical ground reaction force $(\mathrm{N} / \mathrm{kg})$ in respect to all the subjects for sit-to-stand motion.

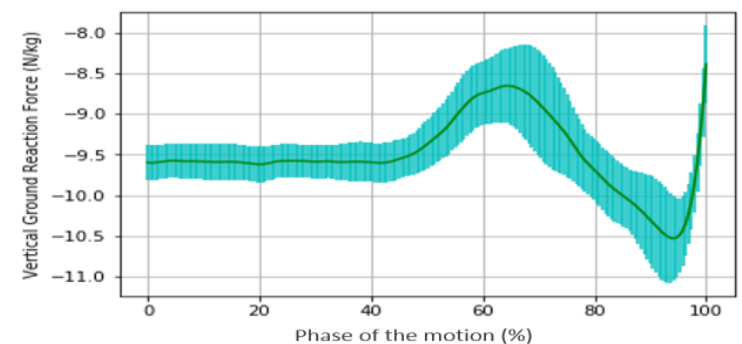

Figure 6. Mean (green line) and standard deviation (light blue) of normalized vertical ground reaction force $(\mathrm{N} / \mathrm{kg})$ in respect to all the subjects for stand-to-sit motion.

noticed, also, in the movement of stand-to-sit and, in this case, the additional force that occurs is lower than in sit-tostand with a difference of approximately $1 \mathrm{~N} / \mathrm{kg}$.

\section{CONCLUSION AND FUTURE WORK}

The results show that the joint angles, the vertical ground reaction force and the joint torques are good descriptors of the sit-to-stand and stand-to-sit movements. Future work includes the use of the acquired data to validate the presented mathematical model and to study control mechanisms to be used in robot assistive devices. Besides that, a study of joint stiffness will be performed using the joint angles and torques.

\section{REFERENCES}

[1] W. Janssen, H. Bussmam and H. J. Stam, "Determinants of the Sit-toStand Movement: A Review," Physical Therapy, vol. 82, pp. 866-879, 2002.

[2] E. A. Chumacero-Polanco and J. Yang, "A review on human motion prediction in sit to stand and lifting tasks," $36^{\text {th }}$ Computers and Information in Engineering Conference, vol. 1A, pp. 1-15, Aug. 2016.

[3] J. Music, R. Kamnik and M. Munih, "Model based inertial sensing of human body motion kinematics in sit-to-stand movement," Simulation Modelling Practice and Theory, vol. 16, pp. 933-944, 2008.

[4] M. Blazkiewicz, I. Wiszomirska and A. Wit, "A new method of determination of phases and symmetry in stand-to-sit-to-stand movement," International Journal of Occupational Medicine and Environmental Health, vol. 27, pp. 660-671, 2014.

[5] A. Mughal and K. Iqbal, "3D bipedal model with holonomic constraints for the de-coupled optimal controller design of the biomechanical sit-to-stand maneuver," Journal of Biomechanical Engineering, vol. 134, pp. 1-9, Apr. 2010.

[6] D. A. Wells, Lagrangian Dynamics. United States of America: McGraw-Hill, 1967, pp. 39-58.

[7] D. A. Winter, Biomechanics and motor control of human movement New Jersey, Hoboken: John Wiley \& Sons, 2009, pp. 82-107.

[8] Vicon Motion Systems Ltd, Plug-in Gait Reference Guide. 2016, pp. 8-14.

[9] V. Shia and R. Bajcsy, "Vision-based Event Detection of the Sit-toStand Transition," ICST Trans. Ambient Systems, vol. 16, pp. 1-4, 2016. 